\title{
MODELIZACIÓN DE LAS RELACIONES ENTRE ESTRATEGIAS DE APRENDIZAJE Y EL RENDIMIENTO UNIVERSITARIO MEDIANTE PREDICCIÓN LOGÍSTICA
}

\author{
ANTONIO HUMBERTO CLOSAS \\ UNIVERSIDAD NACIONAL DEL NORDESTE \\ UNIVERSIDAD TECNOLÓGICA NACIONAL \\ NORBERTO FERREYRA \\ MARIANA VALDÉS \\ MOIRA CARRIÓ \\ DANIELA TORRENTE
}

UNIVERSIDAD NACIONAL DEL NORDESTE ARGENTINA.

“...Ninguna sociedad que olvida el arte de plantear preguntas o que permite que ese arte caiga en desuso puede encontrar respuestas a los problemas que la aquejan...”. Zygmunt Bauman. 


\section{RESUMEN}

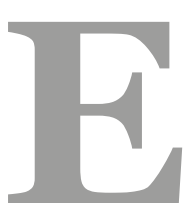

l objetivo principal de este estudio fue desarrollar un modelo de regresión logística que explique de qué manera las estrategias de aprendizaje, que habitualmente utilizan los estudiantes universitarios, se encuentran vinculadas con el rendimiento académico. Concretamente, se examinaron las relaciones existentes entre las dimensiones adquisición, codificación, recuperación y apoyo, correspondientes a la Escala de Estrategias de Aprendizaje ACRA-Abreviada, y las calificaciones obtenidas por los alumnos encuestados, los cuales pertenecen a una Universidad Nacional (de gestión pública) de Argentina. La muestra estuvo conformada por un total de 125 estudiantes (49 mujeres y 76 hombres), con una media de 21.63 años $(D E=3.09)$. La investigación responde a un diseño cuantitativo, descriptivo, explicativo y transversal. Los análisis de datos relativos a estudios descriptivos y psicométricos, permitieron conocer el comportamiento de cada una de las dimensiones de la prueba, así como los índices de consistencia interna de las cuatro subescalas y del instrumento en su conjunto, valores que en todos los casos pueden considerarse adecuados y correctos. Los cálculos de inferencia estadística implementados dieron lugar a determinar que el modelo logístico que mejor se ajusta a los datos muestrales propone como variables explicativas del rendimiento académico: a) las dimensiones codificación y apoyo de la Escala ACRA-Abreviada, y b) el tipo de carrera que estudian los jóvenes encuestados. Los resultados educativos representan un fenómeno multicausal que posee especial relevancia a la hora de implementar decisiones en el ámbito de la política, planificación y gestión educativa, de allí que el presente trabajo conlleva implícitamente verdaderas perspectivas de transferencia.

Palabras clave: estrategias de aprendizaje, rendimiento académico, estudiantes universitarios, regresión logística.

\section{ABSTRACT}

The aim of this study was to develop a logistic regression model to explain how the learning strategies usually used by university students are related to their academic achievement. Actually, the existing relationships between acquisition, encoding, retrieval and support, considered by the Abridged ACRA Scale of Learning Strategies, and the grades obtained by the students who were interviewed at a national public university (state funded) in Argentina were examined. The sample was composed of total of 125 students ( 49 females and 76 males), with a median age of 21.63 years $(S D=3.09)$. This research followed a quantitative, descriptive, explanatory and transversal design. The analysis of the data related to descriptive and psychometric studies allowed us to learn about the performance of each dimension of the test, as well as the internal consistency of the indexes of the four subscales and of the instrument as a whole. These results could be considered appropriate 
and correct in all cases. The statistical inference that was implemented helped to determine that the logistic model that fits best the sample data suggests, as explanatory variables of academic achievement, the following: a) the abridged ACRA scale encoding and support dimensions, and b) the type of course of studies that the interviewed students took up. The educational results data reveal a multi-causal phenomenon that has a special importance when carrying out decisions regarding educational policies, planning and management; therefore, this study brings about real possibilities of potential applications.

Key Words: learning strategies, academic achievement, university students, logistic regression.

\section{INTRODUCCIÓN}

\subsection{PROBLEMÁTICA Y PLANTEAMIENTO DEL ESTUDIO}

Desde hace tiempo, los resultados educativos en los distintos niveles de enseñanza formal constituyen un complejo problema que genera serias preocupaciones en las autoridades y en diferentes sectores sociales de América Latina, así como de distintas regiones, principalmente, occidentales de nuestro planeta.

Indudablemente, en razón que el individuo es producto de factores tanto genéticos como ambientales que inciden de forma diferenciada, el fenómeno de referencia es resultado de un conjunto de determinantes personales que participan teóricamente de acuerdo con el contexto en el que es analizado.

A modo de ejemplo, respecto de lo sostenido, podemos mencionar que en una investigación elaborada por Schiefelbein y Simmons (1981) para países en desarrollo, sobre los determinantes del rendimiento en educación, se plantea que el mismo es una variable multidimensional influenciada por factores familiares, sociales, pedagógicos y por las características del propio sujeto.

En vista de lo señalado, es natural que al intentar analizar cuáles de los factores están modulando y determinando el rendimiento encontrarse con serias dificultades, pues dichos factores causales se encuentran con frecuencia estrechamente relacionados y en complejas interacciones, que resulta sumamente laboriosa su delimitación a efectos de reconocer de qué manera y en qué medida participan.

Sin lugar a duda, el rendimiento académico general y el rendimiento en determinadas áreas disciplinares presentan diversos aspectos en común; no obstante, creemos conveniente que el estudio de este último fenómeno psicoeducativo debe asumir cierta distancia respecto del primero, dado que dispone de elementos propios de relevancia que hacen plausible su tratamiento particular. Así pues, en esta ocasión nos hemos planteado la posibilidad de llevar a cabo el estudio de los resultados académicos en ámbitos cognitivos específicos de las carreras Licenciatura en Administración (LA) y Licenciatura en Economía (LE), que se imparten en la Facultad de 
Ciencias Económicas (FCE) de la Universidad Nacional del Nordeste (UNNE), Argentina.

En el ámbito universitario, en particular, uno de los factores relacionados con la falta de éxito en el logro de los objetivos por parte de los alumnos, no obstante ser una circunstancia multicausal, se encontraría en la formación general que los mismos tienen al iniciar sus estudios, puesto que la diferencia entre los conocimientos que poseen y los que son requeridos en el nivel superior es verdaderamente significativa.

De distintas maneras hemos expresado que el fracaso académico tiene su origen en diversas causas; así entre las variables que según el consenso teórico explican y modulan de modo relevante esta problemática, se encontrarían como determinantes personales: las aptitudes intelectuales (e.g., inteligencia general y razonamiento), distintas formas de autoconcepto (e.g., académico y social), las estrategias de aprendizaje, las metas académicas, algunas características cognitivo-motivacionales (e.g., las actitudes hacia una determinada asignatura) y las capacidades operativas. En tanto que, como factores contextuales podríamos indicar: ciertos aspectos socio-familiares (e.g., rasgos micro y macrosociológicos), determinadas dimensiones del proceso de enseñanza-aprendizaje, así como algunos elementos propios del clima de clase.

De entre este conjunto amplio de factores individuales y ambientales, en este estudio hemos optado por trabajar con las estrategias de aprendizaje, puesto que es una las variables que de forma directa e indirecta mayor incidencia tiene en el rendimiento de los estudiantes universitarios.

Si bien existe una amplia diversidad de definiciones acerca de las estrategias de aprendizaje cognitivas y metacognitivas, muchas de ellas coinciden en señalar dos elementos esenciales: a) implican una secuencia de actividades u operaciones mentales dirigidas a facilitar el aprendizaje, y b) tienen un carácter consciente e intencional en el que están implicados procesos de toma de decisión por parte del estudiante ajustados al objetivo o meta que pretende conseguir.

Es verdad que la búsqueda de soluciones al problema de los bajos resultados trasciende el marco estrictamente académico; no obstante, el aporte de estudios científicos y la realización de "ajustes" desde la órbita de la Educación Superior tienen una relevancia indiscutible. Decididamente, este nivel de enseñanza es uno de los ámbitos naturales para promover y liderar los cambios que lleven a una evolución continua y estrechamente relacionada con los requerimientos del mundo científico y profesional.

En muchas ocasiones, y por diferentes razones, es necesario efectuar algunas modificaciones (e.g., reducción del número de ítems o exclusión ciertas subescalas) en las pruebas estandarizadas que se utilizan para estudiar determinado fenómeno, a fin de adecuarlas a los objetivos de la investigación. En tales casos, los cambios que se realicen deberían implementarse de manera que permitan conservar la estructura y no afecten significativamente la función para las que fueron diseñadas.

Precisamente, una parte del presente estudio se halla relacionado con este último tema, puesto que nuestro propósito inicial estará centrado en estudiar la fiabilidad y la validez predictiva de la Escala de Estrategias de Aprendizaje ACRA-Abreviada (De la Fuente y Justicia, 2003), con el objeto de explicar la variabilidad del rendimiento en estudiantes de Administra- 
ción y Economía, que asisten a la FCE de la UNNE.

El instrumento original pertenece a Román y Gallego (1994) y, de acuerdo con De la Fuente, Soto, Archilla y Justicia (1998), sus ítems poseen una interesante sensibilidad para establecer relaciones entre las técnicas de aprendizaje y el rendimiento académico, así como diferencias cuantitativas en el uso de las mismas según distintas variables, tales como el sexo, la edad o el tipo de carrera que siguen los estudiantes. Esta prueba es frecuentemente empleada en regiones hispanoparlantes, su ámbito de utilización es la educación tanto secundaria como universitaria y su aplicación, dentro de estos niveles, puede tener lugar en contextos naturales de aula por parte del profesor o en contextos extraaulares (consultorio del psicólogo o del pedagogo, laboratorios, aulas especiales, etc.).

No obstante, las bondades que posee el cuestionario primitivo y su amplia utilización, debido a la importante extensión que tiene y al ámbito en el que está prevista su aplicación, nos hemos inclinado por la Escala ACRA-Abreviada, puesto que esta prueba integrada por cuatro subescalas (Adquisición ${ }^{1}$, Codificación ${ }^{2}$, Recuperación ${ }^{3}$ y Apoyo ${ }^{4}$ ), contiene en cada dimensión sólo aquellos ítems que describen las técnicas mayormente usadas por los estudiantes universitarios. De esta manera, es posible contar con un instrumento que, con un formato más breve, resulte ajustado para ser empleado en el nivel académico superior y, desde luego, de utilidad para medir un constructo de innegable incidencia en los resultados académicos de los alumnos.

Los análisis de datos que se realizaran en la etapa empírica de este estudio pertenecen al dominio de la psicometría y de la estadística descriptiva, aunque sólo en un momento inicial, puesto que los cálculos que llevaremos a cabo posteriormente serán de tipo inferenciales. En efecto, los primeros permitirán conocer los índices de consistencia interna de las cuatro subescalas y del instrumento en su conjunto, así como describir el comportamiento estadístico de cada una de las dimensiones de la prueba y del total de la misma. Los segundos, se presume, darán lugar a determinar la ecuación de regresión logística que mejor describa la asociación entre el rendimiento académico (variable explicada), las dimensiones o categorías del cuestionario de medición empleado, y el tipo de carrera o especialidad que siguen los estudiantes encuestados (variables predictoras).

\footnotetext{
${ }^{1}$ En el ámbito de la adquisición se han constatado dos tipos de estrategias de procesamiento: aquellas que favorecen el control o dirección de la atención, y aquellas que optimizan los procesos de repetición.

${ }^{2}$ Codificar en general es traducir a un código y/o de un código. El proceso de codificación se sitúa en la base de los niveles de procesamiento -relativamente profundos- y, de acuerdo con éstos se aproxima más o menos a la comprensión y al significado. ${ }^{3}$ Esta clase de estrategias sirven para manipular (optimizar) los procesos cognitivos de recuerdo de información en la memoria mediante sistemas de búsqueda y/o generación de respuesta.

${ }^{4}$ Ayudan y potencian el rendimiento de las estrategias de adquisición, codificación y recuperación, incrementando la motivación, la autoestima, la atención, entre otros conceptos. Garantizan el clima adecuado para un buen funcionamiento de todo el sistema cognitivo.

${ }^{5}$ Una de las técnicas estadístico-inferenciales más empleadas en la producción científica contemporánea de distintas áreas de conocimiento, especialmente en ciencias sociales y de la salud.
} 


\begin{tabular}{c}
\hline ANTONIO HUMBERTO CLOSAS - NORBERTO FERREYRA - MARIANA VALDÉS - MOIRA CARRIÓ - DANIELA TORRENTE \\
MODELIZACIÓN DE LAS RELACIONES ENTRE STRATEGIAS DE APRENDIZAJE Y EL RENDIMIENTO UNIVERSITARIO... \\
Pags. 72 - 95 \\
\hline
\end{tabular}

En la parte final del primer apartado de este trabajo, no podemos dejar de indicar que ciertamente existe en la literatura especializada abundante información acerca de las estrategias de aprendizaje (definición, naturaleza y clasificación), así como sobre su tradicional relación con los resultados en educación. Sin embargo, extendernos en conceptos teóricos respecto de ambos constructos (estrategias y rendimiento), también sobre el criterio de inclusión de las estrategias como una de las variables explicativas del éxito o fracaso académico, excede completamente los objetivos de este estudio.

\subsection{PROPÓSITOS DEL ESTUDIO}

En vista de las consideraciones efectuadas en el apartado anterior, es posible sostener que en el presente trabajo nos hemos fijado, en principio, la identificación de una escala simplificada, que pueda dar información rápida, concisa y fiable de las estrategias de aprendizaje que utilizan con mayor frecuencia los estudiantes universitarios. Esta percepción es razonable en virtud de la poca cantidad de técnicas que emplean estos alumnos, por lo que con un número sensiblemente menor de ítems tendríamos configurado un instrumento adecuado para, entre otros fines, describir y comprender el perfil general de las conductas de estudio de una determina población estudiantil.

Con el objeto de contar con una prueba que de manera confiable y válida pueda dar respuesta a lo planteado es que, inmediatamente después, nuestra atención se centrará en evaluar el diseño de dicho instrumento a través del análisis de sus propiedades psicométricas (fiabilidad y validez predictiva).

En definitiva, y de manera formal, podemos decir que en este estudio nos hemos propuesto como objetivo principal elaborar un modelo de regresión múltiple que explique de qué manera ciertas estrategias de aprendizaje, que habitualmente utilizan los estudiantes de Administración y Economía, se encuentran relacionadas con el rendimiento académico.

La importancia de llevar adelante esta investigación radica principalmente en observar y analizar los resultados que deriven del estudio y empleo en nuestro ámbito académico de la Escala ACRA-Abreviada, en virtud del contexto social, económico y cultural al que pertenece. Ciertamente, por tratarse de un análisis estadístico aplicado, el fin último será posibilitar la adopción de decisiones o brindar explicaciones, que sean sustentables y resulten razonables, en esta oportunidad, respecto de un fenómeno de especial interés en el ámbito de la política, planificación y gestión educativa, por lo que el presente trabajo conlleva implícitamente verdaderas perspectivas de transferencia. 


\section{MÉTODO}

\subsection{PARTICIPANTES}

Teniendo en cuenta el objetivo que persigue esta investigación, se ha seleccionado una muestra en la cual su unidad se encuentra integrada por la totalidad de los estudiantes que conforman una entidad con definida personalidad como es el grupo-clase. En base este criterio hemos considerado adecuado, luego de estratificar la población (las carreras LA y LE, representaron los estratos), apelar al método de muestreo por conglomerados; el cual se utiliza, siguiendo a Bisquerra (1989), cuando los individuos constituyen agrupaciones naturales, como por ejemplo los alumnos de una clase. Posteriormente, de forma aleatoria fueron elegidos los grupos-clase (conglomerados), que finalmente integraron la muestra con la que se llevo a cabo el estudio.

Resumiendo, podríamos decir que en el procedimiento utilizado para extraer la muestra hemos combinado los métodos estratificado, por conglomerados y aleatorio simple, para identificar y seleccionar las unidades respectivamente.

En concreto, la muestra estuvo compuesta por un total de 125 alumnos (49 mujeres y 76 hombres), de $2^{\circ}$ a $5^{\circ}$ año, lo que Fox (1981) denomina "muestra aceptante", con una media de 21.63 años $(D E=3.09)$. Los estudiantes se hallaban distribuidos en tres grupos-clase del área de Administración, y cuatro grupos-clase del área de Economía; por cierto, ambas licenciaturas forman parte de las carreras que se ofrecen en la FCE de la UNNE.

\subsection{DISEÑO}

En función del objetivo general el diseño de esta investigación es inicialmente de naturaleza no experimental, y en un segundo momento explicativo. Si consideramos como criterio el tipo de información y el modo de recogerla, este estudio es de estilo descriptivo mediante encuesta.

Por otra parte, en atención a la forma de administrar el instrumento de medición, en esta investigación empleamos la técnica del cuestionario. A su vez, si tenemos en cuenta el marco donde se lleva a cabo, estaríamos hablando de una investigación de campo.

Además, en razón de cómo se miden y analizan los datos, es una investigación de línea cuantitativa. Teniendo en cuenta la instancia de recolección de la información, nuestro estudio responde a una estrategia de corte transversal. En virtud del interés por analizar las asociaciones entre las distintas variables que participan, este trabajo es de perfil correlacional.

En líneas generales, desde el ámbito de la confrontación teórica-empírica, podríamos señalar que la investigación responde a un proceso de carácter hipotético-deductivo, puesto que pretendemos comprobar si la conceptualización de la cual partimos se ajusta a la realidad objeto de estudio, a través de la recolección de datos y su posterior análisis estadístico. 


\subsection{PROCEDIMIENTO}

Una vez seleccionada la muestra, la recolección de datos se llevó a cabo, en cada uno de los grupos-clase, en una única instancia, aunque en diferentes momentos temporales. En primer lugar se les informó a los alumnos participantes que la aplicación del instrumento en cuestión, que se realizó en los meses de agosto y septiembre de 2012, respondía a un trabajo de investigación cuyo objetivo consiste en evaluar un cuestionario sobre las estrategias de aprendizaje que más a menudo emplean cuando se encuentran estudiando y la relación que éstas tienen con el rendimiento en las áreas de administración y economía.

También se les indicó sobre la importancia de responder sinceramente a los distintos temas planteados, que sus respuestas tendrán un carácter estrictamente confidencial y que la participación en el estudio era una decisión absolutamente voluntaria. La aplicación la efectuaron los propios profesores, durante el horario académico habitual que los estudiantes tienen asignado para el cursado de las materias que participaron en este trabajo y con el margen de tiempo adecuado en virtud de la prueba utilizada (en promedio 20 minutos).

\subsection{INSTRUMENTOS}

Según ha sido revelado, a efectos de evaluar las estrategias de aprendizaje hemos optado por aplicar el cuestionario propuesto por De la Fuente y Justicia (2003). El instrumento de referencia se elaboró a partir de la selección de todos aquellos ítems de las Escalas ACRA que la mayoría de los alumnos universitarios han informado que habitualmente utilizan mientras están estudiando, en un grado de aceptación bastante-mucho, tomando como punto de corte una puntuación superior al $75 \%$.

En la prueba original, sobre la que ya hicimos referencia en páginas anteriores, los indicadores de fiabilidad y de validez (de contenido, de constructo y predictiva) informados por sus autores, fueron calculados a partir de los datos empíricos recogidos en una muestra de 650 sujetos. Los detalles acerca del estudio de las características psicométricas del instrumento se encuentran disponibles en el manual correspondiente (Román y Gallego, 1994).

A su vez, con la finalidad de obtener el modelo de predicción que mejor explique en nuestro contexto la varianza de los Resultados académicos en las áreas disciplinares de interés, hemos utilizado como variable respuesta las calificaciones alcanzadas por los alumnos encuestados en las evaluaciones parciales de las distintas asignaturas que tomaron parte en este estudio.

La variable aludida, cuyos datos fueron obtenidos a partir de las actas académicas de examen, originalmente era de tipo continua con valores que iban de uno (1) a diez (10). Sin embargo, a fin de trabajar con observaciones más homogéneas, se ha procedido a su recodificación, tras lo cual hemos obtenido una variable (dependiente) de tipo dicotómica con valores: $\mathrm{o}=$ Desaprobado (calificaciones de 1 a 5 ) y 1 = Aprobado (calificaciones de 6 a 10).

En tanto que, de manera específica, las variables predictoras han sido, ciertamente, las 
cuatro dimensiones: Adquisición, Codificación, Recuperación y Apoyo, de la Escala de estrategias de aprendizaje ACRA-Abreviada para alumnos universitarios (variables continuas), así como la Carrera que siguen los estudiantes de la muestra (variable categórica -dummy-, o = LA y 1 = LE). En la creación de variables ficticias, un principio básico indica que son necesarias tantas variables imaginarias distintas como grupos menos uno haya en la variable cualitativa. En nuestro caso, para la variable Carrera, se necesitaría una variable dummy, puesto que sólo existen dos grupos o categorías.

Cabe señalar que se han seleccionado las calificaciones puesto que son, en general, el criterio social y legal del rendimiento en el ámbito de los centros de enseñanza formal. Por otra parte, es el indicador más utilizado en las investigaciones sobre el tema a pesar de la dispersión o falta de consenso de las diferentes instituciones e incluso entre los profesores de una misma institución.

Así pues, en virtud de lo que antecede, se trabajará con fuentes de información primaria y secundaria, de acuerdo con las consideraciones que se realizan de inmediato.

1. Para la recolección de datos primarios se aplicará la Escala ACRA-Abreviada, con el objeto de medir su comportamiento psicométrico y de utilizar las puntuaciones de sus cuatro dimensiones a efectos de desarrollar la ecuación de regresión logística que mejor se ajuste a los datos empíricos. Para esta acción, ciertamente, el investigador se situará en carácter de observador y los alumnos en carácter de informantes.

2. Las fuentes de información de datos secundarios, según se indicó, serán las actas académicas de examen, a partir de las cuales se obtendrán las calificaciones alcanzadas por los estudiantes encuestados en las evaluaciones parciales de las diversas asignaturas y áreas de conocimiento que participan de esta investigación. Estos datos se utilizarán para conformar las variables Rendimiento académico (dependiente) y Carrera (independiente) para emplearlas, conjuntamente con las dimensiones cognitivas y metacognitivas, en la construcción del modelo de predicción logística, objeto principal de esta investigación.

\subsection{ANÁLISIS DE DATOS}

Como podrá comprenderse, lo que se realizó en primer lugar fue la preparación del material (digitalización, impresión y reproducción de copias) relativo al cuestionario abreviado sobre estrategias de aprendizaje. En segundo término, fue construida la base de datos en formato electrónico, a partir de los valores recogidos por la aplicación del citado instrumento y, lógicamente, de otras fuentes primarias y secundarias que proporcionaron información sobre distintas variables (edad, sexo, ciclo de estudio, calificación y carrera) que, de una manera u otra, formaron parte de este estudio.

En cuanto a lo estrictamente inherente a este apartado, se llevaron a cabo diferentes estudios cuantitativos pertenecientes al dominio de la psicometría (correlación dimensión-total y consistencia interna), también de la estadística descriptiva (algunos estadísticos centrales y 
de dispersión) e inferencial (análisis correlacionales y análisis de regresión logística; para las pruebas de hipótesis, como es habitual, utilizamos la medida $p$-valor).

Los diversos tratamientos estadísticos indicados en el párrafo anterior permitieron, por un lado, conocer el comportamiento de cada una de las subescalas de la prueba utilizada y, por otro, determinar la ecuación de predicción que mejor describía la relación entre la variable explicada (Rendimiento académico) y las variables explicativas (las cuatro dimensiones de la Escala ACRAAbreviada y el tipo de carrera que estudian los sujetos participantes). En todos los casos, el procesamiento de los datos fue realizado con ayuda del programa informático SPSS 20.0.

\section{RESULTADOS}

\subsection{ESTUDIO DE LAS DIMENSIONES DEL CUESTIONARIO APLICADO}

Teniendo en cuenta lo expresado en el apartado anterior se presentan de forma sintética los resultados de aquellos indicadores que hemos estimado más convenientes para caracterizar la muestra en el total de la escala y en las cuatro dimensiones que conforman la prueba aplicada. En efecto, en la Tabla 1 pueden apreciarse para cada variable, entre otras medidas, la media, la desviación estándar, la correlación dimensión-total y el coeficiente a de Cronbach.

Los dos primeros estadísticos son de mucha utilidad, no sólo porque la desviación estándar indica cómo se distribuyen los datos alrededor de la media, sino porque para un conjunto de datos normalmente distribuidos, como es el caso de nuestras distintas dimensiones ${ }^{6}$, aproximadamente el 68\% de los valores se encuentran entre la media más/menos una desviación estándar. Asimismo, algo más del 95\% están entre la media más/menos dos desviaciones estándar y prácticamente todos -superan el 99\%- se hallan entre la media más/menos tres desviaciones estándar. En definitiva, cuando se analiza un conjunto de datos numéricos, el conocimiento de estas dos medidas ayuda a resumir y comprender el comportamiento de los datos de la muestra.

El tercero de los cuatro estadísticos mencionados (correlación dimensión-total), recoge el grado de relación que cada una de las categorías posee con el total de la escala, lo que puede considerarse un indicador de su grado de discriminación. La fiabilidad es una de las características fundamentales de un test, una de las forma de evaluarla es a través del cuarto estadístico (coeficiente $\alpha$ de Cronbach), el cual indica la precisión o estabilidad de los resultados; señala la cuantía en que las medidas de la prueba están libres de errores casuales.

Creemos oportuno destacar a continuación algunos aspectos que surgen de la lectura de

${ }^{1}$ El total de los coeficientes de asimetría y curtosis están dentro del intervalo $[-0.5,0.5]$; además, el estadístico KolmogorovSmirnov posee para cada subescala un p-valor mayor a .05, nivel de significación elegido en el test analítico de normalidad. 
los valores que se encuentran en la Tabla 1, obtenidos a partir de los análisis efectuados sobre los datos empíricos.

En efecto, en primer término es importante mencionar que los estadísticos: media, desviación estándar y correlación dimensión-total, relativos a las distintas dimensiones, han resultado muy similares a los valores informados en el trabajo elaborado por De la Fuente y Justicia (2003), lo que era de esperar en atención a que no se realizaron modificaciones de ningún tipo en las preguntas, ni en la estructura de la prueba. Las tres últimas subescalas, Codificación, Recuperación y Apoyo, muestran correlaciones razonables con la puntuación total de la prueba, observándose la más alta en la primera de ellas.

Tabla 1. Estadísticos descriptivos y de fiabilidad de las dimensiones de la prueba

\begin{tabular}{|c|c|c|c|c|c|c|}
\hline Dimensión & $\begin{array}{c}\text { Número } \\
\text { de Ítems }\end{array}$ & $\begin{array}{c}\text { Puntuaciones } \\
\text { directas (PD) }\end{array}$ & Media & $D E$ & $\begin{array}{c}\text { Correlación } \\
\text { dimensión-total }\end{array}$ & $\begin{array}{c}\alpha \text { de Cronbach } \\
\text { sin la dimensión }\end{array}$ \\
\hline $\begin{array}{c}\text { Estrategias de } \\
\text { adquisición }\end{array}$ & 9 & $\begin{array}{c}\text { Mín. }=12 \\
\text { Máx. }=35\end{array}$ & 25.24 & 5.49 & .42 & .64 \\
\hline $\begin{array}{c}\text { Estrategias de } \\
\text { codificación }\end{array}$ & 10 & $\begin{array}{c}\text { Mín. }=17 \\
\text { Máx. }=39\end{array}$ & 29.10 & 4.44 & .62 & .59 \\
\hline $\begin{array}{c}\text { Estrategias de } \\
\text { recuperación }\end{array}$ & 13 & $\begin{array}{c}\text { Mín. }=27 \\
\text { Máx. }=50\end{array}$ & 38.50 & 4.71 & .57 & .61 \\
\hline $\begin{array}{c}\text { Estrategias } \\
\text { de apoyo }\end{array}$ & 21 & $\begin{array}{c}\text { Mín. }=48 \\
\text { Máx. }=83\end{array}$ & 64.82 & 6.98 & .55 & .62 \\
\hline ACRA-Abreviada (53 ítems) PDMín. = 119 PDMáx. $=202 \quad$ Media =157.67 $D E=16.02 \quad \alpha=.71$ \\
\hline
\end{tabular}

Fuente: Elaboración propia.

Por otra parte, los resultados indican que el cuestionario utilizado puede considerarse un instrumento con una fiabilidad aceptable, puesto que el coeficiente alfa encontrado para el total de la prueba (.71) supera el criterio de .70 recomendado (Nunnaly y Bernstein, 1994). Respecto de los indicadores $\alpha$ de Cronbach cuando se excluye la dimensión, podemos señalar que los valores más bajos pertenecen a las dimensiones Codificación, Recuperación y Apoyo, lo que pone en evidencia el aporte relevante que las subescalas mencionadas realizan respecto de la fiabilidad de la prueba, es especial la citada en primer lugar.

Llegados a este punto, se considera conveniente tener presente un detalle que surge de la simple observación de los resultados obtenidos, nos referimos al hecho que la única categoría de la Escala ACRA-Abreviada que al mismo tiempo posee correlación alta con la puntuación total de la prueba y coeficiente alfa de Cronbach bajo cuando se excluye la dimensión, es la denominada Codificación. 


\subsection{ANÁLISIS CORRELACIONALES}

En este apartado llevaremos a cabo análisis correlacionales entre las cuatro dimensiones que integran la prueba (todas variables continuas), así como entre éstas y las variables Rendimiento académico y Carrera (ambas dicotómicas). La primera razón por la que se realizan estos estudios radica en el hecho de que los coeficientes que se obtengan permitirán inicialmente confirmar o no las relaciones que se presumen existen entre las distintas subescalas del cuestionario. El segundo motivo reside en reconocer la presencia de asociaciones estadísticamente significativas entre las distintas dimensiones de la escala aplicada y las dos variables categóricas antes mencionadas, con el fin de visualizar la posible obtención de un modelo explicativo del rendimiento en las áreas disciplinares de interés.

Tabla 2. Matriz de correlaciones

\begin{tabular}{|c|c|c|c|c|c|c|}
\hline & $\begin{array}{l}\text { Estrat. de } \\
\text { adquisición }\end{array}$ & $\begin{array}{l}\text { Estrat. de } \\
\text { codificación }\end{array}$ & $\begin{array}{l}\text { Estrat. de } \\
\text { recuperación }\end{array}$ & $\begin{array}{c}\text { Estrat. de } \\
\text { apoyo }\end{array}$ & $\begin{array}{c}\text { Rendimiento } \\
\text { académico }\end{array}$ & Carrera \\
\hline $\begin{array}{c}\text { Estrat. de } \\
\text { adquisición }\end{array}$ & \multirow[t]{4}{*}{1} & $r=.43^{* *}$ & $r=.22^{*}$ & $r=.22^{*}$ & $n=.15$ & $n=.28 * *$ \\
\hline $\begin{array}{c}\text { Estrat. de } \\
\text { codificación }\end{array}$ & & \multirow[t]{3}{*}{1} & $r=.43^{* *}$ & $r=.46^{* *}$ & $n=.21^{*}$ & $n=.04$ \\
\hline $\begin{array}{l}\text { Estrat. de } \\
\text { recuperación }\end{array}$ & & & \multirow[t]{2}{*}{1} & $r=.53^{* *}$ & \multirow[b]{2}{*}{$n=.18^{*}$} & \multirow{2}{*}{$\begin{array}{l}n=.09 \\
n=.08\end{array}$} \\
\hline $\begin{array}{c}\text { Estrat. de } \\
\text { apoyo }\end{array}$ & & & & 1 & & \\
\hline $\begin{array}{c}\text { Rendimiento } \\
\text { académico }\end{array}$ & & & & & 1 & $\phi=.31^{* *}$ \\
\hline Carrera & & & & & & 1 \\
\hline
\end{tabular}

${ }^{*} p<.05 \quad{ }^{* *} p<.01 \quad$ Fuente: Elaboración propia.

Nota: Para cuantificar el grado de relación lineal entre dos dimensiones de la Escala ACRA-Abreviada (variables continuas) se utilizó el coeficiente de correlación de Pearson. En cambio, para evaluar la asociación entre cada una de las dimensiones de estrategias de aprendizaje y el rendimiento académico (continua vs. dicotómica), empleamos el coeficiente de correlación biserial puntual (Eta). Por último, la relación entre el rendimiento académico y la carrera que siguen los estudiantes (variables dicotómicas), fue medida a través del coeficiente Phi.

De acuerdo con los resultados de la Tabla 2, puede decirse que la totalidad de las dimensiones que componen el cuestionario, como era de esperar, correlacionan de manera positiva y estadísticamente significativa $(r=.22, p<.05 ; r=.43$ a $.53, p<.01)$. No obstante, las relaciones entre los diferentes tipo de estrategias de aprendizaje y el rendimiento académico, si bien son siempre positivas, sólo resultaron significativas las correspondientes a Codificación ( $\eta=$ $.21, p<.05)$ y Apoyo $(\eta=.18, p<.05)$.

El hecho de haber obtenido un estadístico de correlación significativo entre el rendimiento y las estrategias de Codificación, es sin duda una ocurrencia ciertamente alentadora respecto de las posibilidades de lograr el principal objetivo de esta investigación. En efecto, debido al signo positivo de esta asociación, debería interpretarse que aquellos estudiantes que se identifiquen con una correcta y frecuente utilización de este tipo de recursos cognitivos tendrán 
mayores posibilidades de alcanzar un rendimiento de calidad.

Se recuerda, siguiendo a Román y Gallego (1994), que codificar en general es traducir a un código y/o de un código. El proceso de codificación se sitúa en la base de los niveles de procesamiento -relativamente profundos- $\mathrm{y}$, de acuerdo con éstos se aproxima más o menos a la comprensión y al significado. Asimismo, corresponde señalar que la dimensión Codificación coincide, como predictora de la respuesta, con el resultado de otro estudio realizado en la misma dirección y ámbito social de esta investigación.

A su vez, que haya resultado relevante la correspondencia entre el rendimiento y las estrategias de Apoyo debe considerarse una circunstancia verdaderamente interesante, puesto que el papel destacado de las estrategias en la explicación del éxito o fracaso académico ha sido evidenciado en muchos estudios, sobre todo el efecto que tiene el uso de estrategias de la escala de metacognición; esto es, la capacidad de los sujetos para planificar, evaluar y regular su propio proceso de aprendizaje. De hecho, en un trabajo elaborado por De la Fuente, Soto, Archilla y Justicia (1998), estos autores sostienen que se observa un aumento en el rendimiento de los estudiantes de educación superior cuando se les entrena en las estrategias de Apoyo.

A decir verdad, más allá que los coeficientes de correlación obtenidos en general pueden considerarse aceptables, estábamos esperanzados en que los índices entre las distintas categorías de la prueba y los resultados educativos fuesen mejores; sin embargo, esta presunción empíricamente no pudo ser comprobada. Ahora bien, la situación aludida no debería sorprendernos demasiado puesto que, según informan Román y Gallego (1994), en la prueba original la ecuación de predicción, por ejemplo, para la variable respuesta Rendimiento escolar en Ciencias sólo contenía como variable independiente a la dimensión Apoyo $(r=.17, p<.01)$. En general, los distintos modelos que tratan de explicar la varianza de los resultados educativos en determinadas asignaturas, según los autores mencionados, contienen una escala (Recuperación para Lengua y Matemáticas) o dos (Recuperación y Apoyo para Historia) de las cuatro posibles, como predictoras de la variable respuesta.

\subsection{REGRESIÓN LOGÍSTICA}

En vista del objetivo planteado en este estudio y de los coeficientes obtenidos en el análisis correlacional, se ha considerado adecuado emplear en la estimación del modelo de regresión logística el método "Introducir”, el cual es recomendable en aquellos casos que el investigador se halla interesado en conducir el estudio en función de los resultados que va logrando.

Se recuerda, además, que los propósitos del modelo logístico residen en determinar la existencia o ausencia de relación entre una o más variables independientes y la variable dependiente; medir la magnitud de dicha relación y explicar y/o predecir la probabilidad de que la variable criterio sea igual a 1, en función de los valores que adopten las variables predictoras (Jovell, 1995).

Pues bien, en atención a lo expresado, ha sido ingresada como variable respuesta el Ren- 
dimiento académico (de tipo dicotómica, o = Desaprobado y 1 = Aprobado), y como variables explicativas o covariables las dimensiones Codificación y Apoyo de la Escala ACRA-Abreviada (por cierto, ambas de tipo continuo), así como la Carrera que estudian los individuos encuestados (también binomial, o = LA y 1 = LE). En virtud de las opciones elegidas y de los datos de la muestra, el modelo logístico obtenido presenta la siguiente ecuación:

$p($ Rend. académico $=$ Aprobado $)=\frac{1}{1+e^{-(-0,16+0,17 \text { Codificación }-0,07 \text { Apoyo }+1,48 \text { Carrera })}}$

La Tabla 3, muestra los distintos valores estimados para los coeficientes del modelo:

$\mathrm{Bo}=-0.16, \mathrm{~B}_{1}=0.17, \mathrm{~B}_{2}=-0.07 \mathrm{y}_{3}=1.48$; junto con sus respectivos p-valores asociados: .83, .oo, .04 y .oo. Puede observarse que el $p$-valor relativo a cada uno de los coeficientes de las variables predictoras $\mathrm{B}_{1}, \mathrm{~B}_{2} \mathrm{y}_{3}$ es inferior a .05; por lo tanto, para un nivel de significación $\alpha=.05$, se rechaza la hipótesis de que dichos coeficientes son nulos y, en consecuencia, concluimos que las variables asociadas a los mismos: Codificación, Apoyo y Carrera, respectivamente, son relevantes a la hora de explicar el comportamiento de los resultados académicos.

Además de los coeficientes de regresión $\left(\mathrm{B}_{0}, \mathrm{~B}_{1}, \mathrm{~B}_{2}\right.$ y $\left.\mathrm{B}_{3}\right)$ y de sus correspondientes p-valores, la Tabla 3 presenta los respectivos estadísticos de Wald: 0.01, 8.37, 4.02 y 9.86. Teniendo en cuenta el criterio del test de Wald, también podemos asumir en esta ocasión que los coeficientes del modelo son estadísticamente distintos de cero, debido a que para las tres variables predictoras de la respuesta el valor obtenido es superior a 4.

Tabla 3. Coeficientes del modelo y estadísticos de Wald

\begin{tabular}{|c|c|c|}
\hline Variables & $B$ & Wald \\
\hline Constante & -0.16 & 0.01 \\
\hline Codificación & 0.17 & 8.37 \\
\hline Apoyo & -0.07 & 4.02 \\
\hline Carrera & 1.48 & 9.86 \\
\hline
\end{tabular}

Con respecto al contraste para el modelo completo, podemos indicar que el p-valor correspondiente a la prueba Chi-cuadrado (21.71) ha resultado .oo, por lo que se rechaza la hipótesis nula de que todos los coeficientes de las variables incluidas en el modelo son iguales a cero. A su vez, la prueba de Hosmer-Lemeshow, otra forma de evaluar la bondad de ajuste de un modelo de regresión logística, ha proporcionado un $p$-valor de .87, para el estadístico Chi-cuadrado cuyo medida resultó 3.84, de manera que en sintonía con lo expresado en el párrafo anterior, podemos sostener que el modelo que se propone se ajusta a los datos empíricos.

En relación con la capacidad global que el modelo posee para explicar correctamente los resultados del rendimiento académico debemos indicar que es del 72\% (90 aciertos de 125 posibles).

En el afán de ser más específicos, podemos mencionar que el modelo se caracteriza por te- 
ner una baja especificidad (28.95\%) y una alta sensibilidad (90.81\%). Esto significa que clasifica muy bien a los estudiantes con buenos rendimientos académicos (de los 87 alumnos que según la muestra están en el grupo de aprobados, el modelo pronostica exitosamente a 79 de ellos). Sin embargo, no sucede lo mismo a la hora de clasificar a los estudiantes de bajo rendimiento (de acuerdo con los datos de la variable Rendimiento académico, de un total de 38 alumnos desaprobado, el modelo pronostica en esta categoría únicamente a 11 de ellos), siempre considerando que el punto de corte de la probabilidad de la variable dependiente se establece en $50 \%$.

Con el objeto de traducir en forma práctica la utilización del modelo propuesto, se presenta de inmediato la siguiente situación particular. En efecto, si se aplicara la ecuación de regresión a los datos de un estudiante de Economía (se recuerda que en la variable Carrera esta licenciatura toma el valor 1) que tenga, por ejemplo, una puntuación directa superior a la media en las dimensiones Codificación y Apoyo de la Escala ACRA-Abreviada (esto es, mayor a 29.10 y 64.82, respectivamente), digamos 30 y 70, obtendremos:

$$
\begin{aligned}
p(\text { Rend. académico }=\text { Aprobado }) & =\frac{1}{1+e^{-(-0,16+0,17 \cdot 30-0,07 \cdot 70+1,481)}} \\
& =\frac{1}{1+e^{-1.52}}=0.82
\end{aligned}
$$

Es decir, la probabilidad que un alumno, que posea las características indicadas, se encuentre en el grupo de jóvenes que tienen buen rendimiento académico es de un $82 \%$. En otras palabras, puesto que la probabilidad obtenida, por cierto, varía de o a $1 \mathrm{y}$, además, el punto de corte se encuentra establecido en 0.5 , se concluye que al sujeto, cuyos datos hemos utilizado para llevar adelante los cálculos, el modelo lo clasificará como Aprobado. Este resultado es absolutamente razonable en virtud de los valores elegidos en las variables predictoras, especialmente los que corresponden a las subescalas Codificación y Apoyo, dado que para los estudiantes de Economía el valor de la variable Carrera será igual a 1, en todos los casos.

En el contexto de la realidad planteada, así como en virtud de los coeficientes que poseen las escalas Codificación y Apoyo, es posible inferir que cualquier incremento por encima de la media en los niveles de la primera variable predictora (varía de 17 a 39), producirá un efecto de crecimiento sobre la probabilidad de la variable respuesta, independientemente de los valores que asuma la segunda variable (oscila entre 48 y 83). La circunstancia descripta se presenta debido a que, una vez decidido el tipo de licenciatura, se contará con dos términos constantes en la expresión lineal que forma el exponente del número " $e$ " (el primero de ellos es el valor que resulta en el proceso de estimación de los coeficientes de regresión), que podrían operarse conformando de este modo una ecuación particular para cada carrera, tal como puede observarse a continuación.

Así pues, siguiendo a Rosel, Jara y Herrero (2014), dada la transformación de la variable independiente dicotómica en dummy, es posible sostener la existencia de dos ecuaciones de regresión, una para cada grupo o carrera, a partir del modelo logístico general. 
De manera que, la ecuación de predicción para los estudiantes de LE, sería:

$$
p(\text { Rend. académico }=\text { Aprobado })_{L E}=\frac{1}{1+e^{-(-1,32+0,17 \text { Codificación }-0,17 \text { Apoyo })}}
$$

En cambio, la modelización estadística para los alumnos de LA, resulta:

$$
p(\text { Rend. } \text { académico }=\text { Aprobado })_{L A}=\frac{1}{1+e^{-(-0,16+0,17 \text { Codificación }-0,17 \text { Apoyo })}}
$$

De esta manera, ha quedado en evidencia que ambos grupos conservan los coeficientes en las variables explicativas continuas, pero difieren en el término independiente.

Lo expuesto anteriormente revelaría que aquellos estudiantes de Economía que tienen buenos puntajes en las respuestas a los ítems de la dimensión Codificación, no requieren tanto la colaboración de otros procesos de naturaleza metacognitiva (planifican y supervisan la acción de las estrategias cognitivas), como sería el grupo de las estrategias de Apoyo, a efectos de lograr un correcto rendimiento académico.

Por otra parte, es sencillo cuantificar la factibilidad de que el estudiante, cuyos datos estamos analizando, se encuentre en el grupo de Aprobados o en el de Desaprobados, para ello calculamos el odds ratio (OR) asociado al suceso:

$$
O R=e^{1.52}=4,57
$$

Este valor indica que el alumno en cuestión tiene una posibilidad de estar en el grupo de Aprobados 4.57 veces mayor que en el de Desaprobados.

Un análisis similar podría realizarse para los estudiantes de Administración. A modo de ejemplo, podríamos indicar que un alumno de esta área de conocimiento con un valor en la dimensión Codificación de 30 puntos y de 70 en Apoyo (ambas cantidades por encima del promedio), el modelo propuesto lo ubicara en la categoría de Aprobados.

Ahora bien, si la puntuación en la primera escala se mantuviera constante y, en cambio, se incrementara en la segunda variable (significaría que el rendimiento del sistema cognitivo requiere mayor colaboración de otros procesos de naturaleza metacognitiva, oréctica, social, etc.), el modelo de predicción evaluaría a dicho alumno en el grupo de Desaprobados.

Por el contrario, si disminuyera de 70 el valor en la segunda variable (indicaría que el estudiante se encuentra correctamente sensibilizado hacia las tareas de aprendizaje en tres ámbitos: motivación, actitudes y afecto), siempre que en la primera variable mantuviéramos el puntaje igual a 30, la ecuación de regresión clasificaría al estudiante en cuestión en el grupo de Aprobados.

Todas estas apreciaciones, claramente, se realizan en el marco de las posibilidades (fortalezas y debilidades) que el modelo posee para explicar la varianza del rendimiento de los individuos de la muestra, a partir de las puntuaciones en las subescalas que participan y de la carrera que siguen los estudiantes. Por lo tanto, estas estimaciones e interpretaciones deben ser consideradas con debida prudencia, en razón del particular escenario empírico en el que se ha desa- 
rrollado el trabajo y de la metodología de abordaje que ha sido implementada, circunstancias ambas que indudablemente limitan las bondades que la presente investigación pudiera ofrecer.

Sin embargo, en atención a que el número de aciertos globales del modelo es uno de los indicadores más importante de la bondad de ajuste del mismo, podemos sostener que la ecuación estimada resulta razonablemente correcta para clasificar o predecir las categorías de la variable dependiente. En efecto, lo anterior se apoya en el hecho de que si se aplicara el modelo propuesto a las observaciones muestrales, se obtendría un porcentaje de éxitos del $72 \%$, según ha sido anticipado.

Finalizamos esta sección indicando: a) los análisis descriptivos y correlacionales, llevados a cabo en los dos apartados precedentes, proporcionaron información coherente con el modelo logit obtenido; b) la aplicación realizada de la ecuación de regresión muestra que los estudiantes que denotan una utilización más frecuente de las estrategias de Codificación (puntajes altos en esta variables), y pueden prescindir moderadamente de las estrategias de Apoyo (puntajes en torno a la media), tendrían mayores probabilidades de alcanzar los objetivos del curso; c) evidentemente, fue posible apreciar la validez explicativa, predictiva y discriminante respecto del rendimiento académico de la Escala ACRA-Abreviada, en especial de las dimensiones que participan como covariables del modelo.

\section{DISCUSIÓN Y CONCLUSIONES}

En el presente estudio nos habíamos propuesto concretar, en un dominio estadístico de tipo psicométrico, descriptivo e inferencial, la elaboración de un modelo de predicción múltiple que sea de utilidad para explicar la variabilidad del rendimiento académico en función de las dimensiones de la Escala ACRA-Abreviada, en estudiantes de Administración y Economía.

Pues bien, en vista de los resultados que fueron presentados en el apartado anterior, podemos afirmar que los aspectos disciplinares, técnicos y metodológicos, puestos en marcha en el marco de esta investigación, nos permitieron lograr aceptablemente el objetivo planteado y, por ende, confirmar en líneas generales nuestra presunción al respecto.

En efecto, a partir de los estudios iniciales (media, desviación estándar, correlación dimensión-total, consistencia interna) realizados sobre las categorías del cuestionario utilizado, fue posible comprobar que, en principio, la prueba aplicada sería un instrumento fiable para medir las estrategias de aprendizaje que más a menudo utilizan los alumnos universitarios.

Según fue posible observar, en una etapa posterior, situada en el marco de la inferencia estadística, se llevaron adelante análisis correlacionales (mediante coeficientes de Pearson, Eta y Phi) y de regresión logística. La aplicación de esta última técnica del área de análisis multivariante hizo factible la obtención del modelo objeto de interés y, consecuentemente, contrastar la validez predictiva de la Escala ACRA-Abreviada; en virtud de lo cual, nuestra 
opinión en relación con los niveles de discriminación respecto de los resultados educativos es, aunque con cautela, lógicamente favorable.

Esto significa, según nuestra interpretación, que la disminución del número de ítems en el instrumento utilizado no dificulta la identificación de los estudiantes en diferentes grupos de logro académico. Así pues, de acuerdo con nuestros resultados, los alumnos que obtienen buenos rendimientos utilizarían correctamente mayor cantidad de estrategias de aprendizaje (en especial las de Codificación) y no precisarían tanto de recursos metacognitivos (estrategias de Apoyo).

Un aspecto que pensamos conveniente destacar es que, si bien es aceptable la capacidad global que posee el modelo obtenido para explicar correctamente los resultados del rendimiento académico (72\%), consideramos que el porcentaje de aciertos podría mejorar en la medida que se pudiera disponer de un mayor número de dimensiones participando como variables predictoras o, en otras palabras, estadísticamente significativas.

No obstante, es necesario reconocer que la relación de las estrategias con respecto al rendimiento académico no es exclusivamente directa. La capacidad predictiva de esta variable está mediatizada por otras, especialmente de corte motivacional, que ejercen influencia sobre el rendimiento, formando así un entramado de relaciones directas, indirectas y recíprocas.

En un plano más bien complementario al desarrollo efectuado para el presente trabajo, valoramos conveniente señalar que fue posible constatar, mediante análisis multivariado de varianza, que existe distinción por sexo sólo en el uso de estrategias de Adquisición (el p-valor asociado al estadístico $F=7.55$ de contraste resulto menor a .05), por parte de los estudiantes de la muestra (se observó que en promedio las mujeres, $M=26.88$, utilizan con mayor frecuencia que los hombres, $M=\mathbf{2 4 . 1 8}$, este tipo de recursos cognitivos).

Resultados análogos; es decir, diferencias estadísticamente significativas en el uso de acciones y pensamientos que ocurren durante el aprendizaje, en el marco de la dimensión antes referida, se obtuvieron para los factores ciclo de estudio $(F=6.73, p<.05$; la media en estudiantes del ciclo superior, $M=28.88$, es mayor a la media en alumnos del ciclo inicial, $M=24.73$ ), y carrera o especialidad ( $F=10.65, p<.05$; los estudiantes de Administración, $M=26.98$, superan en valores medios a los de Economía, $M=23.87$ ).

En cambio, según nuestros cálculos, la variable independiente edad influye en el uso de estrategias de Apoyo $(F=7.15, p<.05)$. El análisis efectuado indica que en promedio los estudiantes de menor edad (entre 18 y 20 años, $M=66.41$ ) utilizan más a menudo que los alumnos de mayor edad (21 años o más, $M=63.15$ ), aquellas actividades, técnicas y medios que planifican de acuerdo con sus necesidades, a la hora de llevar a cabo los aprendizajes. Posiblemente esta situación sea debido a que los estudiantes más jóvenes, en razón de la falta de experiencia, pueden sentirse inseguros y, en consecuencia, requerir mayor asistencia de procesos de naturaleza metacognitiva.

En estudios de este tipo -empírico, correlacional y no experimental-, los resultados en líneas generales y entre otras causas (e.g., la técnica estadística utilizada para modelizar el fenómeno), dependen de la realidad educativa en la que se lleva a cabo el trabajo de campo, la cual, a su vez, se encuentra influenciada por una serie de factores de origen social, económico y cultural. 
Por esta razón, nuestras apreciaciones pueden servir para el contexto académico en el que se implementó el estudio, también de referencia para investigaciones de similares características a la presente, pero no sería correcto extenderlas a otras áreas, ni comunidades universitarias, mucho menos a otros niveles y sistemas de enseñanza; excepto que se considere que el ámbito de aplicación de los resultados pre-senta cualidad análogas al de esta investigación.

A propósito de las consideraciones que anteceden y a modo de sugerencia, nos permitimos indicar que en futuros trabajos que se efectúen sobre la problemática aquí desarrollada, debido a la riqueza que el tema posee, entre otras cuestiones, podrían tratarse: a) para la escala aplicada, estudio de validez de constructo (mediante el análisis factorial confirmatorio) y de validez externa (a través del análisis multivariado de varianza), y b) utilizando además otros determinantes, elaborar un modelo causal y probar su validez de medida y global, empleando la técnica multivariante denominada estructuras de covarianza.

Como ha sido señalado en diversas ocasiones, el cuestionario que utilizamos sólo contiene aquellos ítems de la prueba original que, según la opinión de un número considerable de alumnos universitarios, utilizan habitualmente mientras están estudiando. Este hecho, evidentemente, genera una pérdida de información producto de la imposibilidad de medir la utilización de otras estrategias y técnicas de aprendizaje que el consenso teórico considera importantes de cara a la evaluación del constructo.

Sin embargo, aún a riesgo de lo mencionado, entendemos que la aplicación realizada es correcta, debido a que tenemos reservas acerca de la conveniencia de evaluar con instrumentos generosos que capten mucha riqueza y profundidad de aprendizaje en ámbitos que no están preparados, que muchas veces son indiferentes y, por tanto, no favorecen el avance de este proceso de tanta trascendencia en todo nivel y sistema educativo.

El trabajo llevado adelante, además de encontrarse en el ámbito del análisis psicométrico, bien podría situarse en el marco de investigaciones de línea cuantitativa que abordan el estudio de fenómenos educativos mediante técnicas estadísticas multivariantes. Por ello, se considera que tanto la temática desarrollada como el tratamiento realizado, constituyen un aporte genuino a la comunidad académica y científica, también a la política, planificación y gestión educativa, en razón de la producción de conocimientos que fue posible generar a partir de datos correspondientes a nuestra región de pertenencia, que no habían sido relevados en trabajos anteriores.

Por último, en sintonía con algunas apreciaciones y sugerencias planteadas en párrafos precedentes, pensamos que sería conveniente que las investigaciones que en adelante se realicen en esta dirección sean contextualizadas, a efectos de contar con nuevos marcos de referencia los cuales permitirían generalizar la aplicación, con ajustes o adaptaciones si fueran necesarias, en el nivel y espacio universitario en el que la prueba objeto de análisis haya sido implementada. 


\section{REFERENCIAS BIBLIOGRÁFICAS}

Bisquerra, R. (1989). Métodos de investigación educativa. Guía práctica. Barcelona: CEAC.

De la Fuente, J. y Justicia, F. (2003). Escala de estrategias de aprendizaje ACRA-Abreviada para alumnos universitarios. Revista electrónica de investigación educativa y psicopedagógica, 1(2), 139-158.

De la Fuente, J., Soto, A., Archilla, I. y Justicia, F. (1998). Factores condicionantes de las estrategias de aprendizaje y del rendimiento académico en alumnos universitarios, a través de las Escalas ACRA. Revista de Educación de la Universidad de Granada, 11, 193-209.

Fox, D. (1981). El proceso de investigación en educación. Pamplona: EUNSA.

Jovell, A. J. (1995). Análisis de Regresión Logística. Madrid: Centro de Investigaciones Sociológicas.

Nunnaly, J. C. y Bernstein, I. H. (1994). Psychometric theory ( $3^{\text {a }}$. ed.). New York: McGraw-Hill.

Román, J. M. y Gallego, S. (1994). Escalas de Estrategias de Aprendizaje, ACRA. Madrid: TEA.

Rosel, J. F., Jara, M. P. y Herrero, F. (2014). Pronóstico con interacción de variables categóricas. Castelló de la Plana, España: Publicacions de la Universitat Jaume I.

Schiefelbein, E. y Simmons, J. (1981). Los determinantes del rendimiento escolar: reseña de la investigación para los países en desarrollo. Bogotá, Colombia: Centro Internacional de Investigaciones para el Desarrollo. 


\section{CURRICULUM VITAE}

\section{Antonio Humberto Closas}

Se desempeña como Profesor Titular de Estadística II y Director de Proyectos de Investigación en la Facultad de Ciencias Económicas de la Universidad Nacional del Nordeste, esta última función también la ejerce en la Facultad Regional Resistencia de la Universidad Tecnológica Nacional. Ha obtenido el grado de Doctor, mención "cum laude", en la Universidad Pública de Navarra, España, en el área de Estadística e Investigación Operativa. Sus líneas de investigación están asociadas con métodos estadísticos multivariantes aplicados a estudios sociales y económicos. Es coautor de libros y de diversas publicaciones en revistas de impacto científico nacionales y extranjeras, algunas de las cuales se encuentran reconocidas en el Social Sciences Citation Index. Es investigador categorizado del Programa de Incentivos, de la Secretaría de Políticas Universitarias, del Ministerio de Educación de la Nación, así como de la Secretaría de Ciencia, Tecnología y Posgrado de la Universidad Tecnológica Nacional. Participa como conferencista y expositor en reuniones académicas que se realizan tanto en Argentina, como en el exterior, relacionadas con su especialidad. Asimismo, es integrante de distintos comités de arbitraje de revistas científicas, también actúa como director o miembro de comisiones de seguimiento y evaluación de tesis de maestrías y doctorados.

hclosas@hotmail.com

\section{Norberto Antonio Ferreyra}

Contador Público. Ha realizado Cursos de Posgrado dictados en la UNNE, tales como "Las Estrategias de Enseñanza en la Universidad”, en 2010, y "Gestión del Capital Humano", módulo correspondiente a la Carrera de Especialización en Gestión Pública Provincial y Mu-

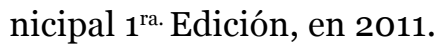

Es Docente Auxiliar de Primera, por concurso, de la Cátedra Principios de Administración, con dedicación simple, y de la Cátedra Organización Administrativa, con dedicación exclusiva, en la Facultad de Ciencias Económicas de la UNNE.

Ha sido integrante de equipos de asistencia técnica como también miembro de numerosos proyectos de investigación acreditados por la Secretaría General de Ciencia y Técnica de la UNNE, habiendo participado de publicaciones, congresos, jornadas y encuentros científicoacadémicos, exponiendo sobre temas relacionados con las Empresas de Familia, su cultura organizacional y rasgos sociológicos de su gestión.

nferreyra@outlook.com 


\section{Mariana Valdés}

Se desempeña como Docente Auxiliar de Primera Categoría en la cátedra Organización Administrativa y Sociología de las Organizaciones, ambas pertenecientes a la carrera de la Licenciatura en Administración de la Facultad de Ciencias Económicas de la Universidad Nacional del Nordeste. En cuanto a su formación en investigación se ha desempeñado como becaria de Iniciación y de Perfeccionamiento de la Secretaría de Ciencia y Técnica de la Universidad Nacional del Nordeste. Ha cursado el Doctorado en Administración en la Facultad de Ciencias Económicas y Estadísticas de la Universidad Nacional de Rosario. Actualmente se encuentra en la ejecución de su tesis.

Es investigadora categorizada del Programa de Incentivos, de la Secretaría de Políticas Universitarias, del Ministerio de Educación de la Nación. También actúa como directora y miembro de tribunal evaluador de tesinas de grado de la Licenciatura en Administración y Licenciatura en Relaciones Laborales.

mvaldes24@hotmail.com

\section{Moira Yanina Carrió}

Se desempeña como Profesor Titular de Principios de Economía y Cunetas Nacionales y Estructura Económica Argentina, también como Subdirectora de Proyectos de Investigación en la Facultad de Ciencias Económicas de la Universidad Nacional del Nordeste. Ha terminado de cursar la Maestría en Economía y Gobierno de la Universal Nacional de San Martin, cuya tesis se encuentra elaborando. Sus líneas de investigación están asociadas con Tópicos referidos a Desarrollo Local, Regional Endógeno. Es autora de diversas publicaciones y exposiciones en Congresos Acreditados. Es investigadora categorizada del Programa de Incentivos, de la Secretaría de Políticas Universitarias, del Ministerio de Educación de la Nación. Participa como conferencista y expositora en reuniones académicas que se realizan tanto en Argentina, como en el exterior, relacionadas con su especialidad. Integra también equipos interdisciplinarios de aplicaciones referidas principalmente al ámbito de las Cuentas Regionales.

mearrio@eco.unne.edu.ar

\section{Daniela Andrea Torrente}

Licenciada en Economía, Especialista en Docencia Universitaria y Maestreando en la Maestría en Gobierno y Economía Política, Escuela de Gobierno Chaco - UNSAM.

Es Profesora Adjunta por concurso de la cátedra Macroeconomía I con dedicación exclusiva en Facultad de Ciencias Económicas de UNNE y es investigadora categoría IV en el Programa de Incentivos. Ha sido Codirectora, Subdirectora e Integrante de diversos Proyectos de Investigación Acreditados, a partir del año 2000 hasta la fecha. Tiene numerosas publicaciones con referato y ha participado en congresos y jornadas vinculados a temas de Economía Regional y 


\section{Formación Docente.}

Se desempeña como Subsecretaria de Extensión y Ejercicio Profesional de la Facultad de Ciencias Económicas, como Directora de la Carrera de Licenciatura en Economía, Codirectora de la Carrera de Especialización en Gestión de la Empresa Agropecuaria (EGEA) y Consejera Superior de la UNNE en representación de la Facultad de Ciencias Económicas.

dtorrente@eco.unne.edu.ar 